Meta

Journal des traducteurs

Translators' Journal

\title{
Les rapports entre la langue et la culture
}

\section{José Mailhot}

Volume 14, numéro 4, décembre 1969

URI : https://id.erudit.org/iderudit/003540ar

DOI : https://doi.org/10.7202/003540ar

Aller au sommaire du numéro

Éditeur(s)

Les Presses de l'Université de Montréal

ISSN

0026-0452 (imprimé)

1492-1421 (numérique)

Découvrir la revue

Citer cet article

Mailhot, J. (1969). Les rapports entre la langue et la culture. Meta, 14(4),

200-206. https://doi.org/10.7202/003540ar d'utilisation que vous pouvez consulter en ligne.

https://apropos.erudit.org/fr/usagers/politique-dutilisation/ 


\section{les rapports}

entre la langue et la culture

Les théories concernant les rapports entre la langue et la culture ont été largement influencées par le développement de la linguistique et de l'anthropologie, plus particulièrement par l'évolution des concepts de langue et de culture. La question des relations entre ces deux phénomènes ne se posait même pas à l'époque où la culture était définie comme un inventaire de traits. Ce n'est qu'à partir du moment où la notion de culture se rapprocha de celle de système et que la langue fut conçue comme intégrée à ce système qu'une partie des conditions nécessaires à la naissance de la question furent réalisées. Une seconde condition était que la langue soit définie comme un fait social. Car ce n'est que dans la mesure où l'on reconnaît que langue et culture constituent des faits de même nature que le problème des relations fonctionnelles entre les deux peut se poser. Toutes ces conditions se réalisèrent il y a à peine plus de trente ans, après la publication des travaux de Durkheim en anthropologie et de Meillet en linguistique.

Faire ici l'exposé et la critique des différentes théories sur la question constitue une tâche complexe en raison du fait que le problème a été posé d'une façon qui varie selon l'acception qu'on donne aux concepts de langue et de culture et qu'en outre les auteurs ont posé le problème des rapports entre les deux à des niveaux fort variés. Au lieu de reconstituer une histoire chronologique des différentes théories, nous procéderons d'après les différentes façons dont le problème a été envisagé dans la littérature.

Déjà au début du siècle, des précurseurs comme Boas et Malinowski furent saisis de la nécessité des études linguistiques en anthropologie. Leur préoccupation se situait d'abord au niveau des rapports pratiques entre une langue et une culture : l'une est-elle nécessaire pour étudier l'autre sur le terrain ? Ils insistèrent tous deux sur l'importance de recueillir le matériel ethnographique en langue indigène. 
Un autre type de question préalable qui a été posée dans la littérature est celle des corrélations entre langue et culture du point de vue de leur distribution. Or il fut très tôt reconnu, par Boas et Sapir notamment, que les cultures et les langues ont souvent des distributions géographiques qui ne coïncident pas. Plusieurs langues génétiquement non reliées peuvent être parlées à l'intérieur d'une aire culturelle homogène et une même langue peut être parlée par des groupes culturellement différents. C'est le cas des langues internationales ou de diffusion et des " langues de marché » comme le swahili en Afrique.

L'aspect de la causalité entre langue et culture a aussi été envisagé. Est-ce la langue qui influence la culture ou est-ce la culture qui influence la langue ? À cela, Boas répond que la forme de la langue est influencée par un certain état de la culture, alors que Whorf met l'accent sur l'aspect limitatif de la langue qui façonne l'expérience. Nous considérons maintenant que c'est mal poser le problème que de le poser en termes de causalité, puisqu'il est admis que ni la langue ni la culture n'est donnée en premier, que les deux sont des phénomènes concomitants et qu'ils s'influencent mutuellement. Nous sommes plutôt à la recherche de corrélations pouvant exister entre les deux qu'à la recherche d'une influence unilatérale.

De quelle façon faudrait-il alors envisager les relations entre langue et culture de manière à dépasser le niveau des questions préliminaires posées par les auteurs du début du siècle? Claude Lévi-Strauss a très bien situé le problème lors d'une conférence réunissant linguistes et anthropologues à Bloomington (Indiana) en 1952:

Pour définir convenablement les relations entre langage et culture, il faut, me semble-t-il, exclure d'emblée deux hypothèses. L'une selon laquelle il ne pourrait $\mathrm{y}$ avoir aucune corrélation entre les deux ordres; et l'hypothèse inverse d'une corrélation totale à tous les niveaux. Dans le premier cas, nous serions confrontés à l'image d'un esprit humain inarticulé et morcelé, divisé en compartiments et en étages entre lesquels toute communication est impossible, situation bien étrange et sans rapport avec ce qu'on constate dans d'autres domaines de la vie psychique. Mais si la correspondance entre la langue et la culture était absolue, les linguistes et les anthropologues s'en seraient déjà aperçu, et nous ne serions pas ici pour en discuter. Mon hypothèse de travail se réclame donc d'une position moyenne: certaines corrélations sont probablement décelables, entre certains aspects et à certains niveaux, et il s'agit pour nous de trouver quels sont ces aspects et où sont ces niveaux ${ }^{1}$.

De multiples études ont été effectuées en tenant compte des différents niveaux de la langue. Leurs résultats sont peu homogènes. Il s'en dégage par conséquent une prépondérance de certains niveaux de la langue quant aux rapports qu'ils peuvent entretenir avec la culture. On admet d'emblée aujourd'hui qu'il n'y a aucune corrélation entre le niveau phonologique d'une langue et la culture où on parle cette langue. Les phonèmes qui sont retenus entre un grand nombre de possibilités théoriques par une culture le sont sur la base d'un choix arbitraire.

1. C. Lévi-Strauss, «Linguistique et anthropologie », Anthropologie structurale, Paris, Plon, 1958 , p. $90-91$. 
Certaines théories, considérées aujourd'hui comme parfaitement farfelues, ont pourtant été formulées, entre autres par Van Gineken qui suggère l'existence d'une corrélation entre les caractéristiques phonétiques d'une langue et des types de culture (tropicale/arctique, paysanne/urbaine). Point n'est besoin d'insister.

L'intérêt pour la morphologie de la langue en tant que susceptible de révéler des corrélations avec des comportements et des attitudes culturelles, a engendré un certain nombre d'études dont les plus célèbres sont celles de Benjamin Lee Whorf. Avant d'en venir aux études plus globales de Whorf, évoquons certains problèmes qui se posent au niveau des corrélations simples entre grammaire et culture.

Faisant fi du caractère purement arbitraire des distinctions morphologiques prônées par un grand nombre de linguistes, certains anthropologues ont cherché des corrélations directes entre un caractère morphologique particulier d'une langue et des attitudes socioculturelles. C'est ainsi que Goodenough, après avoir analysé les différentes formes de possessifs de la langue parlée sur l'île de Truk, dans l'espoir d'y découvrir des reflets du système de propriété, dut conclure qu'une telle analyse linguistique ne révèle rien d'autre que les façons dont on conçoit les rapports entre le sujet et l'objet. De la même façon, Lounsbury, qui recherchait dans les attitudes culturelles envers les femmes oneida une confirmation de l'existence dans leur langue de deux préfixes différents pour le genre féminin, a obtenu des résultats négatifs.

Cette façon de poser le problème rappelle l'explication - trop simple pour être prise au sérieux - qu'ont fournie les missionnaires du dix-neuvième siècle de l'existence dans les langues algonquines de deux catégories grammaticales des noms. Ce qu'ils ont appelé les noms " animés» par opposition aux noms " inanimés » correspondait selon eux aux êtres vivants et aux objets qui ont de l'importance aux yeux des Indiens. Cette conception a été reprise et raffinée par Hallowell, anthropologue intéressé à la vision du monde des Indiens ojibwa. Convaincu qu'il existe un rapport entre le genre des noms et l'« orientation cognitive », il reconstitue toute la métaphysique ojibwa à partir de la notion de personne qui inclut des êtres humains et des êtres autres qu'humains. Ces derniers sont classés dans la catégorie «animés» parce qu'ils détiennent un certain pouvoir et ont la capacité de se métamorphoser.

Les linguistes algonquinistes, dont Hockett, sont en désaccord avec l'explication de Hallowell. Les catégories animés/inanimés qu'ils proposent d'ailleurs d'appeler $A$ et $B$ pour éviter toute confusion, n'ont pas selon eux de valeur sémantique et correspondent à une distinction de caractère purement linguistique, donc arbitraire. Bien que cette théorie soit en général acceptée, Black, au cours d'une recherche sur les classifications indigènes des êtres vivants, a recueilli dans la langue ojibwa quelques cas d'oppositions minimales ayant une correspondance sémantique basée sur le genre. Ces données, bien qu'elles soient insuffisantes pour remettre complètement en question le caractère arbitraire du genre en algonquin, sont à notre avis un indice du fait que la question n'est pas réglée. 
La conception qui constitue le fondement de l'hypothèse whorfienne (à laquelle le nom de Sapir a été plus tard associé) est que l'influence de la langue sur la culture et les activités personnelles réside dans la façon dont elle organise constamment les données de la réalité et analyse les phénomènes. Le monde est en grande partie construit inconsciemment à partir des habitudes linguistiques du groupe. Whorf soutient que l'étude des catégories grammaticales d'une langue peut révéler la métaphysique d'un groupe culturel, c'est-à-dire la façon dont ce groupe organise et segmente l'expérience. Ses études comparées de la langue hopi et des langues S.A.E. (Standard Average European) ont démontré qu'il existe un lien entre, d'une part, la façon dont les deux groupes de langues expriment le nombre, le temps des verbes et désignent les objets physiques et, d'autre part, la façon dont les deux cultures conçoivent le temps, l'espace, la matière, la substance et la forme. Il infère les conceptions culturelles à partir de l'analyse morphologique et confirme ensuite ses hypothèses par l'observation des comportements dans plusieurs domaines de la culture. Il conclut qu'il existe un lien entre une langue et une façon de penser qui elle-même influence le reste de la culture.

Même si les travaux de Whorf ont suscité un grand intérêt dans les milieux anthropologiques et même si l'hypothèse Whorf-Sapir a ouvert la voie à un grand nombre d'études dont certaines sont très récentes, nous ne croyons pas qu'il soit à l'abri des critiques. La plus sévère qu'on puisse formuler c'est que ses démonstrations ne nous convainquent pas toujours. La raison en a été formulée par LéviStrauss qui s'étonne du manque d'exigence de Whorf à l'égard de la culture. Il est un fait que si Whorf traite la langue avec méthode et en fait une analyse qui se situe à un certain niveau d'abstraction, il n'en fait pas autant pour la culture. L'aspect culturel de ses études se situe au niveau d'observations grossières et superficielles et son analyse de la culture est à peine élaborée. On peut aussi mettre en doute la prétention qu'a la méthode de Whorf de révéler «la» métaphysique d'un groupe culturel. Par exemple, Greenberg croit que le peu qu'on puisse inférer au moyen d'une telle démarche ne peut absolument pas constituer quelque chose d'aussi cohérent qu'une vision du monde. Nida, par ailleurs, ne croit pas qu'il soit possible de découvrir les aspects significatifs d'une vision du monde par le biais unique de la structure formelle d'une langue.

Jusqu'à récemment, parmi les recherches concernant les rapports langue/culture, un grand nombre s'est concentré sur l'étude du contenu du vocabulaire. La principale conclusion de ces études - que personne ne remet en question - est qu'il y a une corrélation entre le contenu du lexique et les intérêts culturels d'une société. Déjà, Sapir écrivait: «... language is a complex inventory of all the ideas, interests and occupations that take up the attention of the community ${ }^{2} »$. Il est tout à fait évident qu'une langue reflète la culture au sein de laquelle elle est parlée. C'est ce qui explique que les populations arctiques n'ont pas de termes pour désigner les plantes tropicales et que, par contre, elles en possèdent plusieurs pour parler de la neige ou de l'animal qui constitue la base de leur alimentation. Le lien qui existe à ce niveau entre contenu lexical et contenu culturel ne prouve rien d'autre que le fait qu'une langue s'inscrive dans un contexte culturel.

2. P. Penle, éd., Language, Thought and Culture, Ann Harbor, 1958, p. 5. 
Un autre ordre de problèmes a été posé au niveau lexical qui, sans aucun doute, présente plus d'intérêt scientifique que le précédent. Il s'agit de la pauvreté de certaines langues dites primitives en termes abstraits et génériques, indice à partir duquel Lévi-Bruhl conclut à l'existence de la mentalité prélogique. Ce genre de raisonnement a toujours été énergiquement combattu par les Américains en vertu de leur valorisation bien connue de l'indigène. La réaction de Boas à l'idée de mentalité prélogique fut catégorique: si l'Indien nord-américain ne possède pas de terme unique pour désigner l'idée de «pitié » et s'il accole toujours un possessif au concept de «mère », ce n'est pas parce que sa langue est incapable d'exprimer des idées abstraites, c'est que sa culture n'exige pas qu'il exprime des idées abstraites. Le sémanticien Ullmann soupçonne ce genre de raisonnement de cacher un refus d'admettre qu'il $\mathrm{y}$ aurait des langues primitives tout comme il y a des cultures primitives.

Quant à nous, devant cette confrontation des langues prétendues concrètes et des langues prétendues abstraites, nous croyons que la question est surtout de savoir si on n'a pas conclu un peu rapidement à la pauvreté de certaines langues en termes génériques, en se méprenant justement sur le sens de «terme générique ». Il ne nous semble pas que le fait pour une langue de désigner un objet en lui accolant un possessif soit une preuve suffisante. Les tenants de la mentalité prélogique n'ont-ils pas oublié de mentionner que les langues dites primitives, tout comme les autres langues, contiennent tout un inventaire de termes non spécifiques qui correspondent aux catégories d'un univers classifié ? Le terme "plante» par exemple se présente dans plusieurs langues comme un terme générique pouvant inclure d'autres termes plus spécifiques comme «fleur» et «arbre ». Nous croyons que c'est ce genre d'erreur qui a fait conclure à la mentalité prélogique. Sans prétendre régler la polémique, nous voulons indiquer qu'à notre avis le problème initial a été fort mal posé en vertu d'un manque de données adéquates qu'une simple analyse hyponymique faite dans des langues dites primitives suffirait à combler.

Depuis une dizaine d'années, les études lexicales ont été polarisées non pas sur le contenu uniquement mais sur la structure du vocabulaire. Il est maintenant admis que c'est le niveau sémantique de la langue qui se prête le mieux à des inférences concernant la culture, parce que les référents impliqués dans la signification sont des événements non linguistiques. L'école qui est responsable de cette conception des rapports entre langue et culture est l'école américaine d'ethnoscience, appelée aussi ethnographie sémantique, dont les principaux représentants sont Sturtevant, Frake, Pike et Conklin. Cette école a une certaine parenté avec l'école française de sémantique structurale représentée par Greimas et Pottier. Les Américains sont cependant davantage orientés vers des études dintérêt anthropologique que linguistique.

Le concept le plus utile qui a été développé et appliqué par ces deux écoles est celui de champ sémantique:

... lexical fields are highly organized and integrated conceptual spheres whose elements mutually delimit each other and derive their significance from the 
system as a whole. In each field, a sphere of experience, concrete or abstract, is analyzed, divided up and classified in a unique way which embodies a scale of values and a particular vision of the world ${ }^{3}$.

L'école américaine, à qui doit être attribué un grand nombre d'études de champs sémantiques dans d'autres cultures que la nôtre, redéfinit la culture comme étant l'ensemble des classifications indigènes d'une société ou encore comme les façons utilisées par une société pour classifier son univers matériel et social. Jusqu'ici la recherche en ethnoscience a été polarisée sur un certain type de systèmes de classification dont les contours sont relativement faciles à délimiter: la nomenclature des couleurs, la terminologie de parenté, les taxonomies végétales, animales et médicales.

Les deux hypothèses de départ de l'ethnographie sémantique sont que l'esprit humain tend à classer les choses et que s'il classe les choses, il faut aussi, pour qu'elles soient communicables à l'intérieur d'une même culture, que ces classifications figurent dans la langue. Un lexème est alors considéré comme l'étiquette d'une catégorie classificatoire et le vocabulaire relié à un champ sémantique donné, comme un ensemble de termes dont certains sont spécifiques et d'autres génériques (leur nombre dépend de la «profondeur » de la taxonomie) dont il s'agit de reconstituer la structure en niveaux d'opposition. La phase la plus révélatrice de l'opération ethnoscientifique est l'analyse componentielle qui est appliquée successivement à l'ensemble des termes de chaque niveau d'opposition de la taxonomie. Étant une adaptation de l'analyse sémique linguistique à ce genre d'études, elle a pour but de découvrir, par le moyen de différentes méthodes très précises qu'il serait trop long d'exposer, quels sont les critères de regroupement et de différenciation appliqués par les indigènes dans leurs classifications.

Cette approche a déjà fait la preuve qu'elle est extrêmement fructueuse. Elle permet de dégager, par le biais d'une analyse de la structure sémantique de la langue, une façon particulière de voir l'univers dans une culture donnée qui, autrement, demeurerait implicite. Les réserves qu'on peut formuler à l'égard de l'ethnoscience concerneraient ses possibilités d'application à tous les domaines de la culture et donc à la culture comme système. Pourrait-elle révéler les conceptions qui se cachent derrière le vocabulaire religieux par exemple? Et même si cela était, n'y a-t-il pas autre chose dans la culture qu'un ensemble de systèmes de classifications? Il reste malgré tout que le niveau des rapports entre langue et culture qui est envisagé par l'ethnoscience apporte plus, en profondeur et en intérêt scientifique, que toutes les autres conceptions exposées jusqu'ici.

Parmi toutes les façons de concevoir les rapports entre langue et culture, il en est une qui dépasse en promesses toutes les autres en raison du niveau global où elle situe le problème. Il s'agit de celle de l'école structuraliste française dont les idées commencent à être partagées par quelques Américains, dont Goodenough et McQuown. Ces vues nouvelles, dont le grand promoteur est Lévi-Strauss, nous rassurent par leur façon vraiment scientifique de poser enfin le problème des rapports fondamentaux entre la langue et la culture.

3. S. Ullmann, "Semantic Universals ", in Joseph H. Greenberg, éd., Universals of Language, Cambridge (Mass.), M.I.T. Press, 1966, p. 250. 
Les deux phénomènes ne sont pas ici découpés en niveaux mais sont considérés globalement, en tant que systèmes: «... langue et culture sont deux modalités parallèles d'une activité fondamentale: je pense ici à cet être présent parmi nous, bien que nul n'ait songé à l'inviter à nos débats: l'esprit humain ${ }^{4} \gg$. La fonction essentielle des deux systèmes parallèles se résout à l'échange, à la communication. Dans cette ligne de pensée, Goodenough réduit langue et culture à tout ce qu'un individu doit savoir ou croire de manière à fonctionner d'une façon qui soit acceptable à ses congénères. La grande richesse de cette approche réside dans l'analogie - une analogie de structure évidemment - qu'elle établit entre la langue et la culture: «... la culture possède une architecture similaire à celle du langage. L'un et l'autre s'édifient au moyen d'oppositions et de corréla'ions, autrement dit, de relations logiques ${ }^{5} \gg$.

D'après ces conceptions, idéalement tous les domaines de la culture devraient pouvoir être considérés sous l'angle de systèmes de relations. Il a été brillamment démontré par Lévi-Strauss que cela est possible pour la structure sociale et les systèmes de parenté, pour l'art et la mythologie. Il faudrait, bien sûr, qu'un inventaire complet de la culture soit entrepris dans la perspective de la méthode structuraliste pour nous convaincre définitivement que langue et culture sont les deux produits parallèles de l'activité inconsciente de l'esprit humain.

Au terme du long et ardu développement historique des rapports entre langue et culture, les deux seules conceptions qui, à notre avis, méritent encore qu'on s'y arrête sont celles de l'école structuraliste française et de l'école américaine d'ethnoscience. Le grand mérite scientifique de la première réside dans son postulat de l'unité de l'esprit humain, postulat qui fait déboucher la recherche anthropologique sur des préoccupations plus globales et plus passionnantes que jamais. Quant à la deuxième, elle met l'accent sur la diversité des réalisations concrètes de l'esprit humain dans les différentes cultures, en postulant aussi que l'esprit de l'homme fonctionne d'une façon cohérente et que sa préoccupation essentielle est d'ordonner les choses en les opposant. Ces deux écoles nous semblent ouvrir des voies extrêmement prometteuses pour la recherche future.

JosÉ MAILhot

4. C. Lévi-Strauss, "Linguistique et anthropologie», Anthropologie structurale, Paris, Plon, 1958, p. 81 .

5. Ibid., p. 79 . 